\title{
Usages de poudres à base de rhizomes de Cochlospermum tinctorium Perrier ex A.Rich au Bénin : fréquence, formes et indications
}

\author{
Appolinaire Kouamé DOSSA ${ }^{1}$, Jean Robert KLOTOE ${ }^{1,2^{*}}$, Eric AGBODJENTO', \\ Victorien DOUGNON ${ }^{1}$, Julien SASSA SINKOU ${ }^{3}$ et Frédéric LOKO ${ }^{1}$
}

\author{
${ }^{1}$ Unité de Recherche en Microbiologie Appliquée et Pharmacologie des substances naturelles (URMAPha), \\ Laboratoire de Recherche en Biologie Appliquée (LARBA), Ecole Polytechnique d'Abomey-Calavi (EPAC), \\ Université d'Abomey-Calavi, 01BP2009 Cotonou, Bénin. \\ ${ }^{2}$ Ecole Normale Supérieure de Natitingou ; Université Nationale des Sciences, Technologie, Ingénierie et \\ Mathématiques; BP72 Natitingou. \\ ${ }^{3}$ Association Nationale des Praticiens de la Médecine Traditionnelle du Bénin (Anaprametrab). \\ *Auteur correspondant, E-mail: jrklotoe@yahoo.fr
}

Received: 12-06-2021 $\quad$ Accepted: 25-08-2021 $\quad$ Published: 31-08-2021

\section{RESUME}

Cochlospermum tinctorium est une plante médicinale utilisée dans plusieurs pays pour le traitement de l'hépatite. Au Bénin, différentes poudres à base de ses rhizomes sont vendues dans les marchés et utilisées par les tradithérapeutes pour plusieurs usages. L'objectif de cette étude était de répertorier les différentes formes et les divers usages des recettes à base de Cochlospermum tinctorium utilisées au Bénin. Dans ce sens, une enquête ethnomédicinale a été menée auprès de 434 usagers des marchés et 40 tradithérapeutes du Sud et du Nord Bénin suivant la méthode par interview semi-structurée. Les données obtenues ont montré que les recettes à base de $C$. tinctorium sont plus connues et utilisées au Nord-Bénin. Ces recettes se présentent sous trois formes (A, B et C). Leurs usages sont alimentaires (41\%) et médicinales (59\%). La forme A est très utilisée (93,91\%) par la population enquêtée pour les besoins nutritionnels et thérapeutiques alors que les formes $\mathrm{B}$ et $\mathrm{C}$ ont, essentiellement, un usage médicinal. Cette étude a permis de comprendre la diversité de recettes associées au terme "poudre jaune" au Bénin. Il est dès lors nécessaire d'évaluer la toxicité et l'efficacité de ces différentes formes d'usage de la poudre de rhizome de $C$. tinctorium.

(C) 2021 International Formulae Group. All rights reserved.

Mots clés : Bussoromou, poudre jaune, usages endogènes.

\section{Uses of rhizome-based powder of Cochlospermum tinctorium Perrier ex A.Rich in Benin: frequency, forms and indications}

\begin{abstract}
Cochlospermum tinctorium Perrier is a medicinal plant used in several countries for the treatment of hepatitis. In Benin, different powders made from its rhizomes are sold in markets and used by traditional healers for several purposes. The objective of this study was to list the different forms and uses of Cochlospermum tinctorium-based recipes used in Benin. An ethnomedicinal survey was conducted among 434 market users and
\end{abstract}


40 traditional healers from Southern and Northern Benin using the semi-structured interview method. The data obtained showed that the recipes based on C. tinctorium are more known and used in North Benin. These recipes come in three forms (A, B and C). Their uses are food (41\%) and medicinal (59\%). Form A is widely used $(93.91 \%)$ by the population surveyed for nutritional and therapeutic needs while forms B and C have, essentially, a medicinal use. This study has allowed us to understand the diversity of recipes associated with the term "yellow powder" in Benin. It is therefore necessary to evaluate the toxicity and effectiveness of these different forms of use of $C$. tinctorium rhizome powder.

(C) 2021 International Formulae Group. All rights reserved.

Keywords: Bussoromou, yellow powder, endogenous uses.

\section{INTRODUCTION}

Les plantes médicinales sont utilisées continuellement pour les besoins de santé primaire par une majeure partie de la population des pays en voie de développement. L'Organisation Mondiale de la Santé (OMS) confirme cette pratique médicale et rapporte que plus de $80 \%$ de la population de ces pays ont recours à la médecine traditionnelle basée sur l'utilisation des plantes médicinales (OMS, 2002). Dans les pharmacopées africaines, une variété d'espèces végétales est utilisée dans la préparation des recettes médicinales employéés dans la prise en charge traditionnelle de diverses affections.

Cochlospermum tinctorium Perrier ex A.Rich. (C. tinctorium) est une plante médicinale appartenant à la famille des Bixaceae (The Plant List, 2012). C'est un sousarbrisseau (plante ligneuse à la base et dont les ramifications sont herbacées) atteignant $80 \mathrm{~cm}$ de haut, à rhizome souterrain ligneux produisant des pousses annuelles. Ses feuilles sont alternes, palmatilobées, stipules linéaires et caduques (Abdulaziz et al., 2019). En médecine traditionnelle, les vertus médicinales de différentes parties de C. tinctorium, particulièrement de son rhizome, sont reconnues dans plusieurs pharmacopées africaines (Ballin et al., 2002). En Afrique de l'Ouest, le rhizome de $C$. tinctorium est utilisé dans le traitement de la jaunisse et de maladies de foie (Ahmad et al., 2021). Il sert aussi à soigner les œdèmes, l'incontinence urinaire, la dysménorrhée, l'épilepsie, la schistosomose, la pneumonie, les affections bronchiques, la conjonctivite, les problèmes gastriques, la diarrhée, l'indigestion, les maux d'estomac et les affections de la peau (Ahmad et al., 2021).
Au Burkina Faso, des extraits de rhizome de la plante sont indiqués dans le traitement de la malaria (Zerbo et al., 2007). Au Nigeria, une recette à base de ses fruits et de tamarin est indiquée pour soigner les morsures de serpent (Igoli et al., 2004). Le rhizome quant à lui est utilisé dans le traitement de la lèpre (Maiha et al., 2009). En Côte d'Ivoire, la pulpe des feuilles est utilisée comme pansement humide pour faire mûrir les abcès et les furoncles alors que le décocté des rameaux ou de rhizome est efficace dans le traitement des troubles génitourinaires, des douleurs rénales ou intercostales (Ballin et al., 2002). Au Mali, la plante s'utilise dans le traitement des maladies gastro intestinales, de la jaunice, de la malaria et de la schistosomose (Nergard et al., 2005).

Au Bénin, très peu de données ethnomédicinales existent, à l'étape actuelle, sur les usages traditionnels de la poudre jaune issue du rhizome de Cochlospermum tinctorium Perrier ex A.Rich. La présente étude vise donc à répertorier les différents formes et usages des recettes à base de Cochlospermum tinctorium utilisées au Bénin.

\section{MATERIEL ET METHODES Milieu d'étude}

L'étude s'est déroulée dans les grandes villes du Nord-Bénin (Kandi, Parakou, Natitingou et Djougou) et du Sud-Bénin (Cotonou et Abomey-Calavi). La région du Sud Bénin est située entre $6^{\circ} 25 \mathrm{~N}$ et $7^{\circ} 30^{\prime} \mathrm{N}$ et couvre une superficie de $17109 \mathrm{~km}^{2}$. Le climat est de type subéquatorial caractérisé par un régime pluviométrique bimodal avec deux saisons pluvieuses alternées par deux saisons sèches. La température moyenne annuelle est de $28{ }^{\circ} \mathrm{C}$ et l'humidité de l'air varie entre $69 \%$ 
et 97\% (Akoègninou, 2004). Les sols dominants sont les sols ferralitiques sur sédiments argileux, les sols hydromorphes dans les vallées, les bas-fonds et les plaines alluviales, les vertisols dans la dépression de la Lama et les sols bruns eutrophes tropicaux (Igue et al., 2013). Elle appartient à la zone guinéo-congolaise qui comprend une mosaïque d'îlots de forêts denses humides, de savanes, de prairies, de mangrove et de jachères. Il y a été recensé 1170 espèces végétales (Adomou et al., 2011). Sa population compte 5.369 .774 habitants avec une densité variant de 100 habitants $/ \mathrm{km}^{2}$ en général à 322 habitants $/ \mathrm{km}^{2}$ dans l'Atlantique. Les groupes ethniques qui dominent sont les Fon et apparentés $(39,2 \%)$, les Adja et apparentés $(15,2 \%)$ et les Yoruba et apparentés (14,5\%) (INSAE, 2013). Les activités économiques dominantes sont le commerce et l'agriculture. On y pratique aussi les cultures maraîchères, l'élevage, la pêche et l'artisanat.

Au Nord Bénin, la population de Kandi est estimée à 179290 Habitants ; 103843 habitants à Natitingou ; 255478 habitants pour Parakou et 255478 habitants pour Djougou (INSAE, 2013). Les groupes ethniques les plus représentés sont les Bariba (38\%), Peulh (22\%), Dendi (21\%), Mokolé (12\%), Gando (7\%) (INSAE, 2013). La savane arborée ou arbuste dans les régions soudanaises du Centre et du Nord abrite une faune riche et diversifiée. Dans cette partie du Bénin, le climat est de type tropical avec une saison sèche qui s'étend de novembre à mai et une saison des pluies qui s'étend de juin à septembre (Akoègninou, 2004). Pendant la saison sèche les températures atteignent $40^{\circ} \mathrm{C}$ et le harmattan souffle avec un air sec et chargé de poussières. Parmi les principales activités figure l'agriculture employant $42 \%$ de la population (culture du sorgho, du maïs, du riz et surtout du coton). Elle est suivie de l'élevage qui emploie 33\% de la population. Les autres activités secondaires sont le commerce et l'artisanat.

\section{Matériel}

Le matériel utilisé dans le cadre de la présente étude était constitué d'un questionnaire d'enquête, d'un appareil photo numérique, d'une balance de précision et d'un appareil audio pour enregistrer les interviews.

\section{Méthodes \\ Collecte des données}

Il s'est agi d'une étude transversale menée dans les villes de Kandi, Parakou, Natitingou, Djougou, Cotonou et AbomeyCalavi. Ces villes représentent les plus peuplées du Nord et du Sud Bénin. Les cibles enquêtées sont les usagers des marchés de Calavi kpota, Cocotomey, Godomey pour la commune d'Abomey-Calavi; de Vêdoko et Tokpa pour la commune de Cotonou; de Azèkè pour la ville de Parakou, du marché central pour la ville de Kandi, le marché Yara pour la ville de Natitingou et le grand marché pour la commune de Djougou. Des informations complémentaires ont été recherchées chez quelques tradithérapeutes de ces villes. L'enquête menée par la méthode par interviews semi-structurées s'est déroulée durant la période allant du 07 février au 30 août 2020 ( Klotoé et al., 2013; Agbodjento et al., 2020). Les informations renseignées étaient le sexe, l'âge, l'ethnie, les zones de récolte de la plante de Cochlospermum tinctorium, les différentes formes de présentation des recettes à base de Cochlospermum tinctorium, les diverses sources d'approvisionnement, les motifs d'utilisation et les indications thérapeutiques.

\section{Analyse des données}

Les données socio-démographiques des enquêtés ont été analysées par une statistique descriptive à l'aide du tableur Excel. Les variables quantitatives ont été exprimées en moyenne \pm écart type. La fréquence de citation (FC) a été utilisée comme indice ethnobotanique pour analyser les données relatives aux usages endogènes (traitement des maladies et symptômes de maladies).

FC

$=\frac{\text { Nombre d'enquêtés ayant cité la maladie }}{\text { Nombre total d'enquêtés }} \times 100$

Les critères descriptibles de la norme de présentation des produits pharmaceutiques et alimentaires de FAO-WHO (2020) ont été 
utilisés pour caractériser les différentes formes de recettes recensées dans cette étude.

\section{RESULTATS \\ Caractéristiques socio démographiques des enquêtés et usages endogènes}

L'étude a porté sur 434 usagers des marchés et 40 tradithérapeutes. $49,31 \%$ des enquêtés étaient de sexe masculin et 50,69\% étaient de sexe féminin. Au Sud-Bénin (Cotonou et Abomey-Calavi), les enquêtés ont déclaré n'avoir pas connaissance des usages endogènes de la poudre jaune à base du rhizome de Cochlospermum tinctorium. Par contre, au Nord-Bénin, 45,62\% des sujets enquêtés ont déclaré avoir connaissance des recettes à base de Cochlospermum tinctorium. $66,96 \%$ des femmes et $57,83 \%$ des hommes avaient au moins une fois utilisés ces recettes (Tableau 1).

Les utilisateurs de la poudre jaune à base de Cochlospermum tinctorium enquêtés dans cette étude ont différents niveaux d'instruction. Près de la moitié d'entre eux sont analphabètes $(48 \%) ; 29 \%$ ont fait les études primaires ; $17 \%$ les études secondaires et $6 \%$ les études universitaires. Par ailleurs, les caractéristiques relatives à l'ethnie des enquêtés sont présentées par la Figure 1. De cette figure, il ressort que les ethnies les plus représentées étaient les Waama $(21,74 \%)$, Otamari $(17,39 \%)$, Naténi $(16,52 \%)$ et Batombou (13,04\%).

Les données recueillies concernant l'âge des utilisateurs de la poudre jaune sont résumées au niveau de la Figure 2. L'analyse de ces données révèle que l'effectif des utilisateurs varie proportionnellement suivant l'âge. Les enquêtés âgés de 40 ans et plus utilisaient majoritairement les recettes à base de Cochlospermum tinctorium.

Par ailleurs, les enquêtés utilisent la poudre jaune à base de Cochlospermum tinctorium pour trois motifs. La moitié de ceuxci employait les différentes formes de la poudre jaune recensées à des fins de traitement des affections, $6 \%$ les utilisent pour la prévention des affections et $41 \%$ pour des raisons d'ordre nutritionnels. Concernant les indications thérapeutiques, il est à noter que la poudre jaune à base de Cochlospermum tinctorium est utilisée dans le traitement des affections hépatiques $(55,65 \%)$, du paludisme $(51,30 \%)$, de l'ictère $(44,35)$ (Figure 3). Des indications thérapeutiques similaires ont été renseignées par les tradithérapeutes (Figure 4).

\section{Zones de récolte de C. tinctorium au Bénin}

Au Bénin, l'habitat naturel de la plante de C. tinctorium est situé au Nord-Bénin précisément dans les communes de Malanville (Goungou), Toucountouna (Kouarfa), Kouandé (Guilimaro), Natitingou (Perma), Tchaourou (Bétérou, Kika) Kérou, Ouassa Pehonco, Nikki, Banikoara, Copargo, Kalalé et Pèrèrè (Figure 5).

Méthodes courantes de préparation de la poudre jaune

Les rhizomes de Cochlospermum tinctorium récoltés sont lavés puis rincés à l'eau de pompe. Ensuite, ils sont épluchés pour obtenir la matrice pure (la chaire) qui poursuit le processus de transformation en poudre jaune. La matrice ainsi obtenue subit un broyage partiel (l'émiettement) donnant ainsi un produit avec beaucoup de fibres. Séché au soleil pendant des jours, le broyat sec est pilé et/ou moulu. La poudre obtenue est ensuite tamisée à l'aide d'un tamis à petites mailles pour obtenir de la poudre fine appelée poudre jaune (Figure 6).

\section{Caractéristiques des recettes à base de $C$. tinctorium vendues au Nord-Bénin}

Les données recueillies ont permis l'identification de trois formes de recettes à base de Cochlospermum tinctorium nommées A, B, et C (Figure 7 ; Tableau 2). Il existe deux types de la forme A : A1 et A2. Les formes B et $\mathrm{C}$ sont les formes améliorées de la forme traditionnelle (forme A). Elles sont exclusivement utilisées à des fins thérapeutiques. La forme A (Bussoromou) possède un double usage à savoir nutritionnel et thérapeutique. Les enquêtés qui utilisent la forme A représentent $93,91 \%$ et ceux utilisant les formes $\mathrm{B}$ et $\mathrm{C}$ représentent respectivement $1,74 \%$ et $4,35 \%$. Les utilisateurs de la forme $\mathrm{A}$ ont déclaré que la poudre est utilisée pour cuisiner la viande de chien dans le but de la garnir et la rendre plus attrayante. Elle est utilisée dans l'intention d'anéantir les toxines 
supposées être présentes dans cette viande selon la croyance populaire. Dans la ville de Natitingou, la poudre est utilisée comme ingrédient de base pour préparer la sauce par toutes les ethnies. Les autres appellations traditionnelles du Bussoromou à Natitingou sont Kpaararou (en Bariba), Kota ou Kpata (en Deendi), Dimonsonri (en Ditamari), Soonri (en Naténi). Les formes B et C sont utilisées principalement à but thérapeutique (curatif et préventif).
Le Tableau 3 résume les données issues de l'appréciation des différentes formes de recettes à base de Cochlospermum tinctorium selon les normes de présentation des produits pharmaceutiques et alimentaires. De ce tableau, il ressort que les formes $\mathrm{B}$ et $\mathrm{C}$ ont un fort taux de conformité supérieure à la forme A. Cependant d'une façon générale les taux de conformité de ces produits restent faibles si on considère les normes de présentation des produits pharmaceutiques et alimentaires.

Tableau 1: Répartition des enquêtés selon le sexe, la connaissance et l'utilisation des recettes à base de $C$. tinctorium.

\begin{tabular}{lccc}
\hline Variables & Catégories & Total & Pourcentage \\
\hline Sexe & Masculin & 214 & $49,31 \%$ \\
\cline { 2 - 4 } & Féminin & 220 & $50,69 \%$ \\
\hline Connaissance des recettes & Masculin & 83 & $38,79 \%$ \\
\cline { 2 - 4 } médicinales à base $\boldsymbol{C}$. tinctorium & Féminin & 115 & $52,27 \%$ \\
\hline Utilisateurs de Bussoromou & Masculin & 48 & $57,83 \%$ \\
\cline { 2 - 4 } & Féminin & 77 & $66,96 \%$ \\
\hline
\end{tabular}

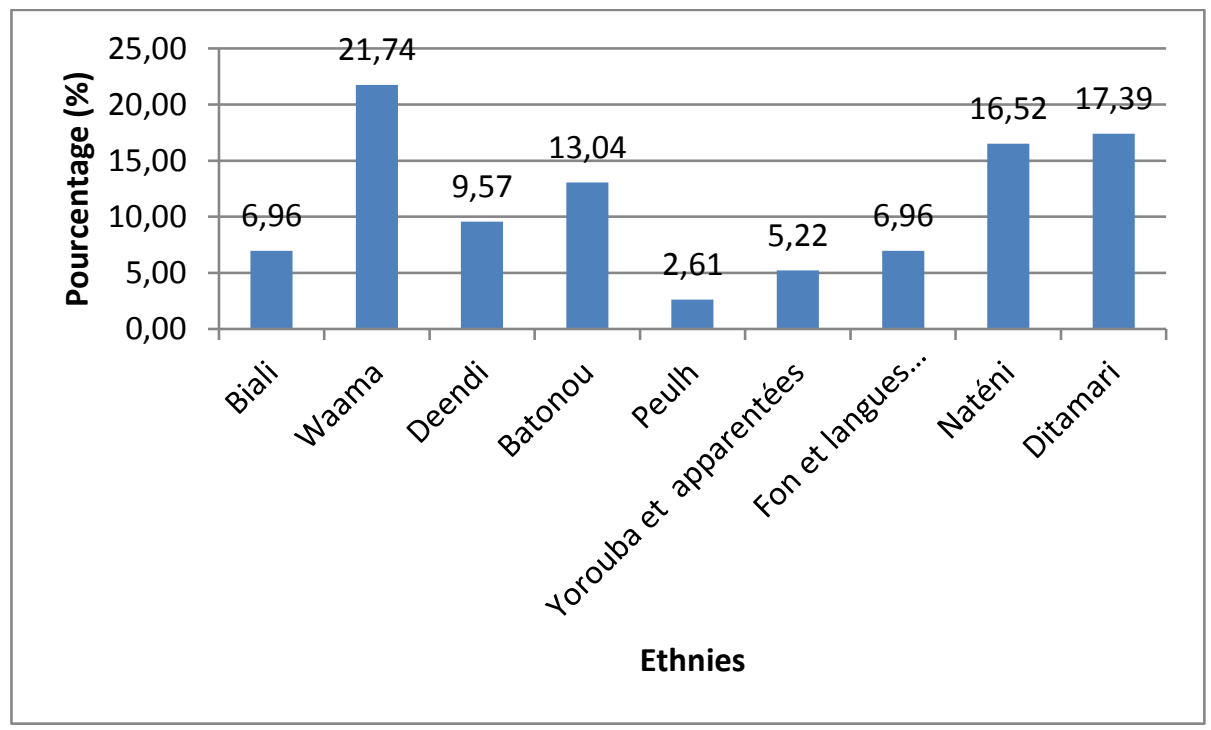

Figure 1 : Répartition des utilisateurs des recettes à base de Cochlospermum tinctorium selon l'ethnie au Nord-Bénin. 
Tableau 2 : Description des échantillons des recettes à base de la poudre de rhizome de $C$. tinctorium vendues au Nord Bénin.

\begin{tabular}{|c|c|c|c|c|}
\hline & Forme A1 & Forme A2 & Forme B & Forme C \\
\hline Conditionnement & sachet & sachet & sachet & Boîte en plastique \\
\hline Lieu de vente & marché & marché & $\begin{array}{l}\text { Place croix rouge } \\
\text { (boutique de } \\
\text { vente des produits } \\
\text { médicinaux) }\end{array}$ & $\begin{array}{l}\text { Hôpital St Jean de Dieu } \\
\text { de Tanguiéta et } \\
\text { annexes (Bakhita...) }\end{array}$ \\
\hline $\begin{array}{l}\text { Prix de vente (en } \\
\text { FCFA) }\end{array}$ & 25 & 25 & 2000 & 3000 \\
\hline Couleur & Jaune-orangée & Jaune pâle & Jaune pâle & Jaune pâle \\
\hline Composant & $\mathrm{RCt}+$ B.karité & $\mathrm{RCt}$ & $\mathrm{RCt}+C$. odorata & $\mathrm{RCt}+C$. micranthum \\
\hline Poids moyen & 104 & 97 & 117 & 95 \\
\hline Indication & $\begin{array}{l}\text { médical et } \\
\text { alimentaire }\end{array}$ & $\begin{array}{l}\text { médical et } \\
\text { alimentaire }\end{array}$ & médical & médical \\
\hline $\begin{array}{l}\text { Quelques } \\
\text { appellations }\end{array}$ & $\begin{array}{l}\text { poudre jaune et } \\
\text { Bussoromou }\end{array}$ & $\begin{array}{l}\text { poudre } \\
\text { jaune et } \\
\text { Bussoromou }\end{array}$ & $\begin{array}{l}\text { poudre jaune et } \\
\text { Bussoromou }\end{array}$ & poudre jaune \\
\hline
\end{tabular}

$\mathrm{RCt}=$ poudre du rhizome de Cochlospermum tinctorium ; B.Karité = beurre de karité ; . odorata $=$ Chromolaena odorata et C. micranthum=Combretum micranthum

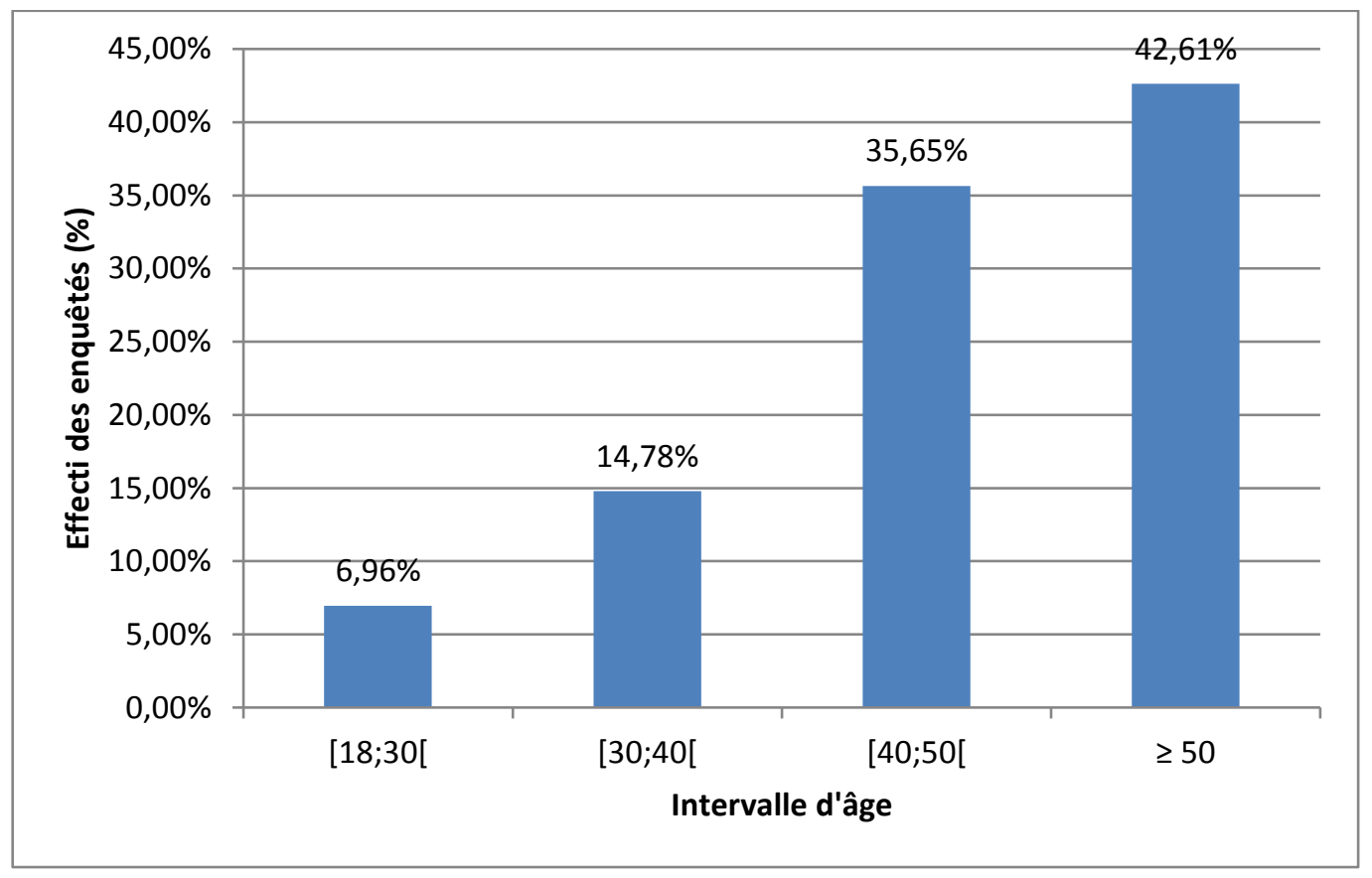

Figure 2 : Répartition des utilisateurs des recettes à base de Cochlospermum tinctorium selon les tranches d'âge. 


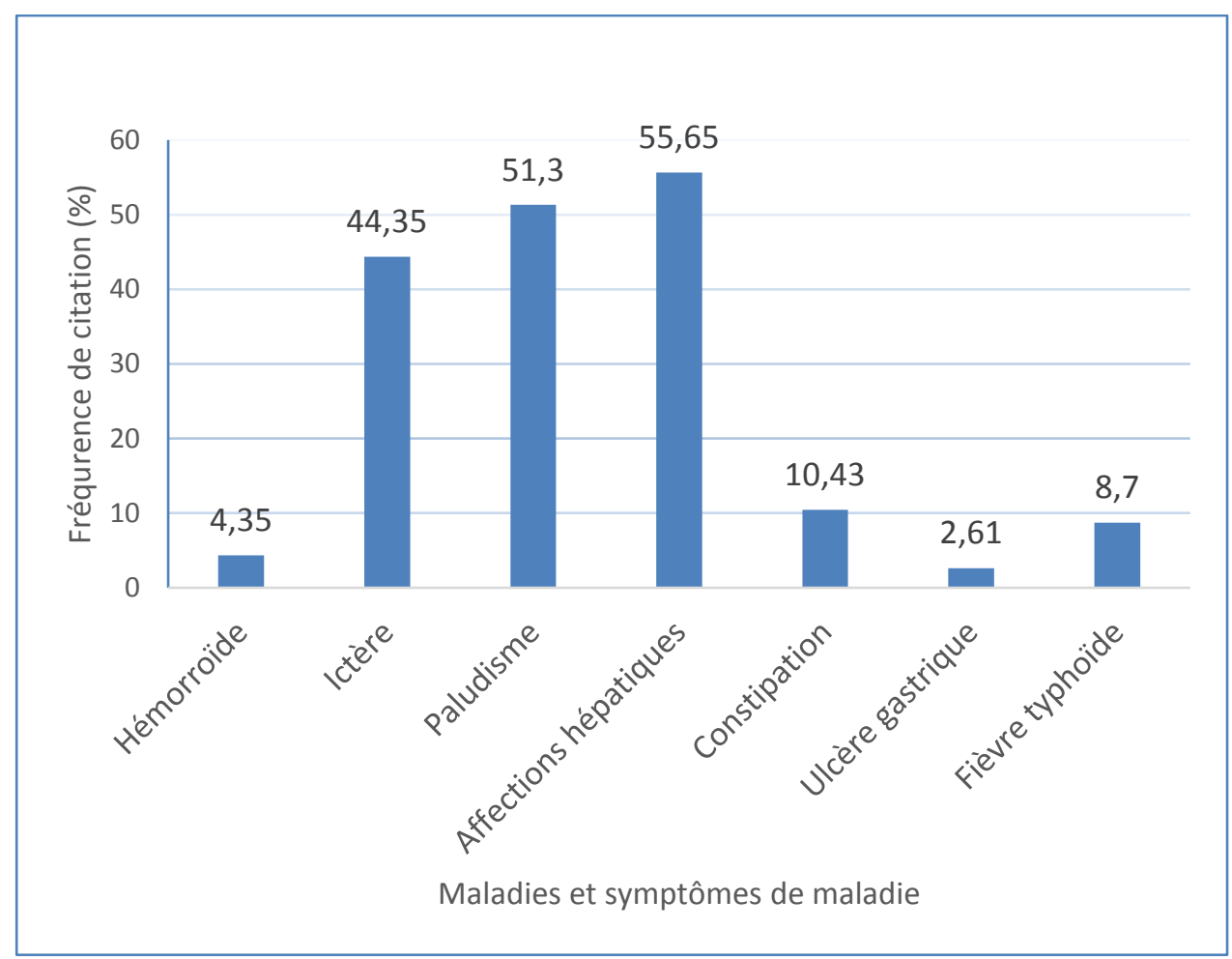

Figure 3: Maladies et symptômes de maladies traités par les recettes à base de Cochlospermum tinctorium selon les utilisateurs.

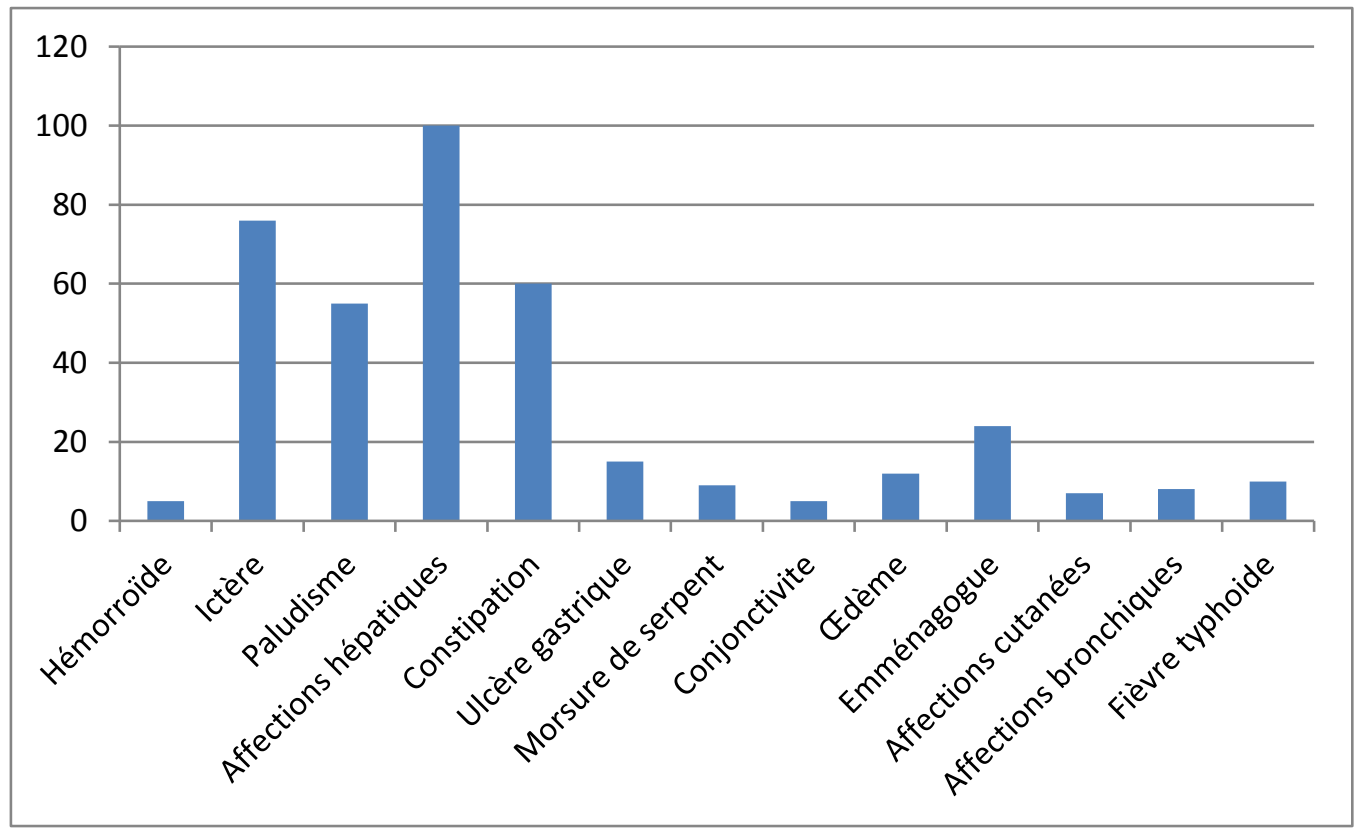

Figure 4: Les différentes affections traitées par la poudre jaune à base de Cochlospermum tinctorium par les tradithérapeutes. 


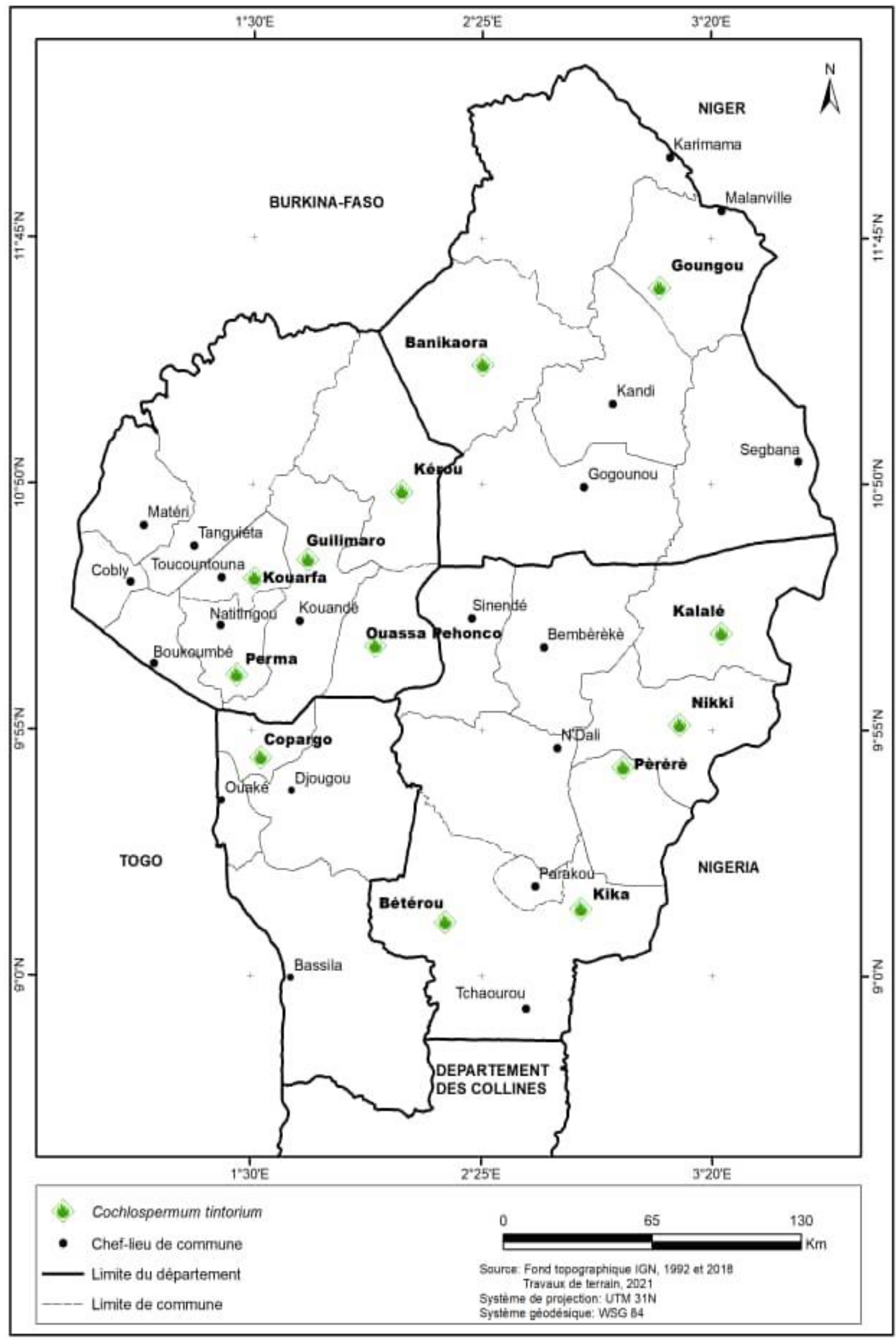

Figure 5 : Carte montrant les zones de collecte de Cochlospermum tinctorium au Bénin. 


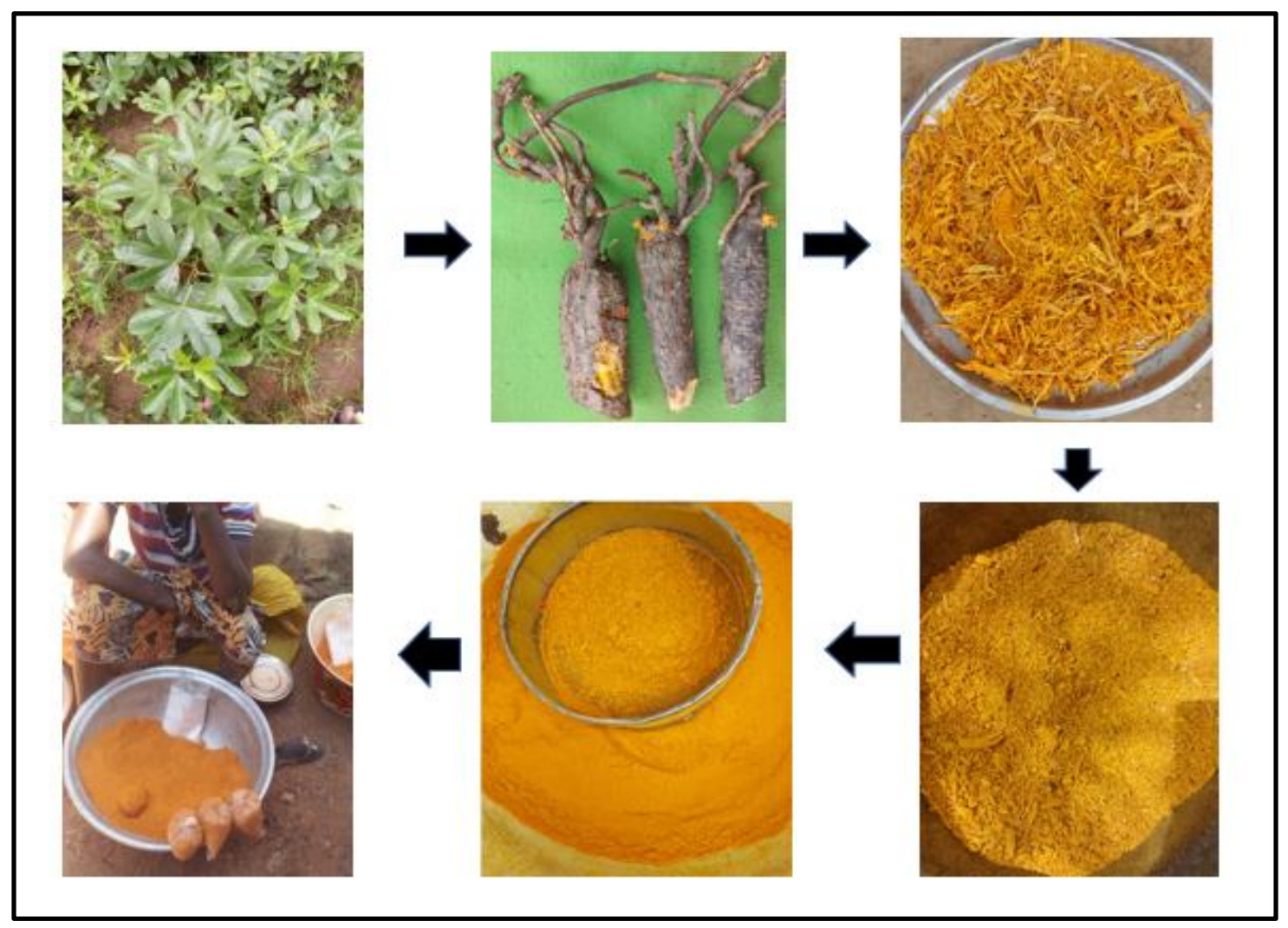

Figure 6 : Illustration de la préparation de la poudre jaune.
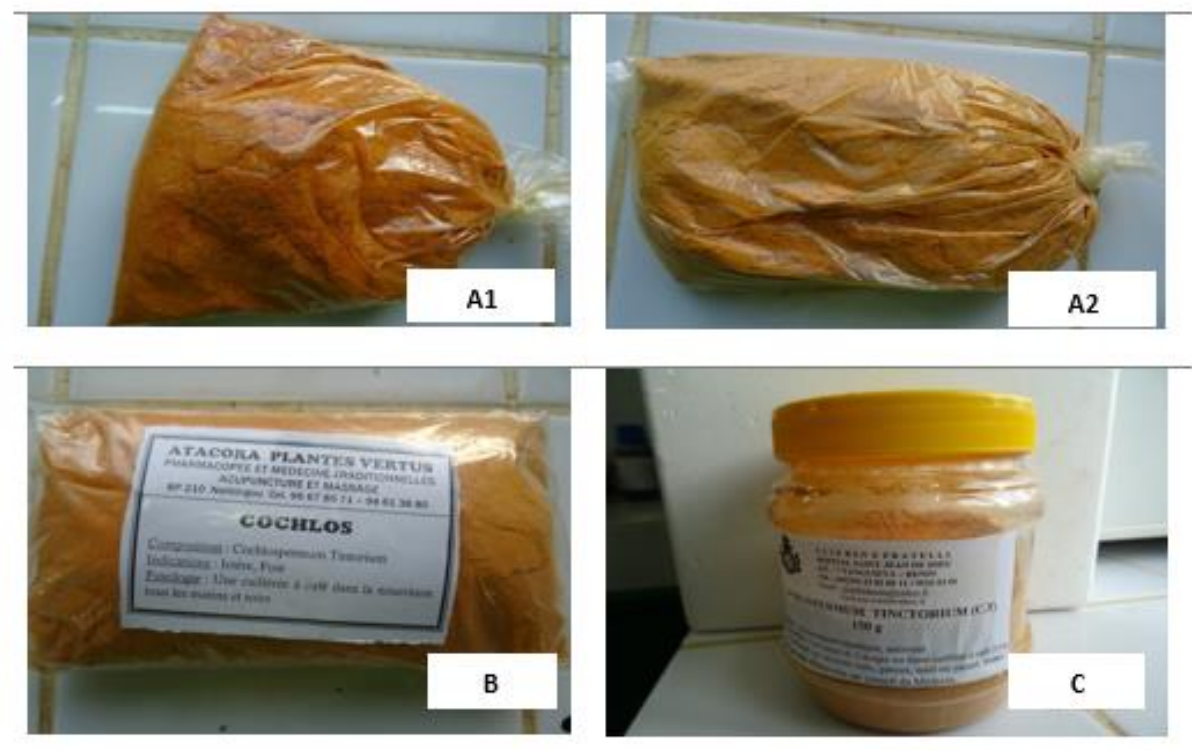

Figure 7 : Photos des recettes à base de Cochlospermum tinctorium vendues au Nord-Bénin. 
Tableau 3 : Appréciation des échantillons des formulations de $C$. tinctorium vendus au Nord Bénin selon la norme Codex.

\begin{tabular}{|c|c|c|c|c|}
\hline Critères & Forme A1 & Forme A2 & Forme B & Forme C \\
\hline Inscription du nom du produit & - & - & + & + \\
\hline $\begin{array}{l}\text { Inscription du nom et adresse du } \\
\text { fabricant }\end{array}$ & - & - & + & + \\
\hline Inscription du poids net sur l'étiquette & - & - & - & + \\
\hline $\begin{array}{l}\text { Conformité entre le poids net inscrit et } \\
\text { le poids net réel }\end{array}$ & - & - & - & - \\
\hline Emballage propre et sec & + & + & + & + \\
\hline $\begin{array}{l}\text { Emballage opaque ne laissant pas } \\
\text { passer la lumière }\end{array}$ & - & - & - & - \\
\hline Emballage hermétiquement fermé & - & - & - & - \\
\hline $\begin{array}{l}\text { Précision de la composition sur } \\
\text { l'étiquette }\end{array}$ & - & - & - & - \\
\hline $\begin{array}{l}\text { Mention de la posologie et du mode } \\
\text { d'emploi }\end{array}$ & - & - & + & + \\
\hline Contre-indication & - & - & - & - \\
\hline Effet secondaire & - & - & - & - \\
\hline $\begin{array}{l}\text { Mention des vertus et des indications } \\
\text { thérapeutiques }\end{array}$ & - & - & + & + \\
\hline $\begin{array}{l}\text { Mention des instructions de } \\
\text { conservation }\end{array}$ & - & - & - & - \\
\hline $\begin{array}{l}\text { Mention de la date de } \\
\text { conditionnement }\end{array}$ & - & - & - & - \\
\hline Mention de la date limite d'utilisation & - & - & - & - \\
\hline Mention du lot de fabrication & - & - & - & - \\
\hline $\begin{array}{l}\text { Taux de conformité globale avec les } \\
\text { normes de présentation }\end{array}$ & 6,25 & 6,25 & 31,25 & 37,50 \\
\hline
\end{tabular}

\section{DISCUSSION}

Cette étude visait à recenser les connaissances relatives aux utilisations endogènes de la poudre jaune à base de Cochlospermum tinctorium au Bénin à l'échelle des usagers des marchés et de quelques tradithérapeutes. Les données collectées renseignent une utilisation exclusive des recettes à base de Cochlospermum tinctorium au Nord-Bénin. Cette observation pourrait s'expliquer par le faite que l'habitat naturel de la plante de $C$. tinctorium au Bénin est situé au Nord du Bénin. Les utilisateurs de ces recettes au Nord-Bénin dans leur majorité les emploient pour des fins médicinales et culinaires sans distinction d'âge, d'ethnie, du niveau d'étude et de sexe. Ces observations confirment que la poudre jaune à base de Cochlospermum tinctorium est bien connue au Nord Bénin. Par ailleurs, les tradithérapeutes enquêtés dans cette région du Bénin ont renseigné que la source de leur connaissance provient de l'héritage ancestral et de l'apprentissage sur le tas. Des observations similaires ont été rapportées par Tabuti et al. (2003) sur l'usage des plantes médicinales. Ces données traduisent que l'entourage et l'expérience des ascendants restent donc le moyen le plus efficace de transmission des connaissances sur les vertus des recettes à base de Cochlospermum tinctorium. Cette idée corrobore celle de Hachi et al. (2015) selon laquelle l'entourage, l'expérience et les herboristes sont les moteurs de la transmission 
intergénérationnelle des savoirs ethnomédicinaux.

Par ailleurs, l'usage des recettes à base de $C$. tinctorium est plus répandu chez les femmes que chez l'homme. Ce constat fait penser que la connaissance médicinale des recettes à base de Cochlospermum tinctorium se transmet des mères aux filles à travers les recettes culinaires et autres activités féminines. Cette observation est aussi conforme à celle de El Yahyaoui et al. (2015) qui ont relevé dans leur étude que les femmes détiennent non seulement la majorité des savoirs culinaires mais aussi des connaissances sur les vertus des plantes médicinales. L'utilisation des trois types de recettes par toutes les ethnies présentes dans le milieu d'étude évoque le caractère non restreint d'usage à un groupe particulier d'ethnie. Ceci confirme le fait que la majeure partie des enquêtés reconnaissent et utilisent les trois types de recettes à base de Cochlospermum tinctorium. Dans cette étude, il est à signaler qu'une faible proportion des jeunes enquêtés (moins de 40 ans) utilise ces recettes. Cette observation traduit que l'usage de ces recettes est délaissé par cette couche de la population en raison de l'influence probable de la modernisation. Ceci évoque le désintérêt de cette couche de la population pour les produits provenant de la médecine traditionnelle. Plusieurs études scientifiques ont souligné ce manque d'intérêt de la jeunesse pour les pratiques endogènes (Mehdioui and Kahouadji, 2007; Benkhnigue et al., 2010; Agbodjento et al., 2020).

Par ailleurs, les données recueillies sur les recettes à base de Cochlospermum tinctorium renseignent que la forme $\mathrm{A}$ (traditionnelle) est la plus consommée. Ceci serait dû à l'ancienneté de son existence, à sa double utilité (alimentaire et médicinale) et à son faible coût de vente. Par ailleurs, les emballages utilisés pour le conditionnement de la poudre de cette forme A ne sont pas opaques et ne permettent donc pas d'assurer une bonne conservation des composants de cette poudre. De plus, il est à signaler l'absence de mention d'instruction de conservation sur les emballages. Cela traduit que les fabricants ne maîtrisent pas l'influence des facteurs qui pourraient altérer la qualité de leur produit. Des données similaires ont été rapportées par Aissi et al. (2013) après une étude sur les poudres des feuilles de Moringa oleifera. Pour ces auteurs, les poudres des feuilles de Moringa oleifera exposées aux rayonnements solaires dans les marchés présenteraient un risque d'altération des principaux composés phytochimiques. Ils appuient cette idée et rapportent que certains éléments nutritionnels et médicinaux sont thermolabiles, sensibles aux rayons du soleil et aussi à la lumière du néon c'est le cas des vitamines $\mathrm{C}$, $\mathrm{A}$ et des $\beta$-carotènes.

Par ailleurs, aucune des formes des recettes à base de Cochlospermum tinctorium vendues au Nord-Bénin n'a présenté un emballage hermétiquement fermé. Cela expose les poudres jaunes à une éventuelle contamination secondaire aux microorganismes ambiants. Il peut exister aussi un risque de pénétration d'air humide pouvant favoriser la péremption prématurée du produit. L'absence d'une date de péremption constatée sur les divers produits peut amener les utilisateurs à consommer des poudres avariées. Les utilisateurs peuvent être ainsi exposés à de probables intoxications alimentaires.

Concernant les usages endogènes de la poudre jaune à base de Cochlospermum tinctorium recensées dans cette étude, il est à préciser que la poudre est utilisée pour les besoins nutritionnels, la prévention et le traitement traditionnel de différentes pathologies. De ce fait, la poudre jaune est utilisée par la population enquêtée comme un alicament. Son usage dans le traitement du paludisme et des hépatites; des affections courantes au Nord Bénin, le désigne comme un produit d'une grande importance pour la population d'étude. Nos observations corroborent les travaux de Akpemi (2012), qui ont souligné la forte utilisation de cette plante dans le traitement des maladies hépatiques. Ces observations sont corrélées avec les propriétés hépato-protectrices des principaux constituants (Cochlospermum tinctorium, Chromolaena odorata et Combretum micranthum) des trois formes de recettes recensées ( Nergard et al., 2005; Mouzouvi et al., 2014; Favi et al., 2020) 


\section{Conclusion}

Les recettes à base de Cochlospernum tinctorium sont très utilisées par la population enquêtée au Nord-Bénin. La forme A est la plus utilisée. Elle est largement utilisée par la population enquêtée pour des fins médicinales et culinaires sans mention des données sur leur degré de toxicité et d'efficacité. L'exploration des potentiels thérapeutiques et du degré de toxicité de ces recettes à base de Cochlospernum tinctorium revêt donc une importance capitale pour sa valorisation et la garantie de sa sécurité d'utilisation.

\section{CONFLITS D'INTERETS}

Il n'y a pas de conflit d'intérêts en rapport avec cet article.

\section{CONTRIBUTIONS DES AUTEURS}

AKD et JRK ont participé à la conception de l'étude. AKD, EA et JSS ont effectué la collecte et l'analyse des données. $\mathrm{AKD}, \mathrm{JRK}$ et EA ont participé à la rédaction du manuscrit. VD et FL ont assuré la direction scientifique de l'étude. Tous les auteurs ont lu et approuvé le travail.

\section{REMERCIEMENTS}

Les auteurs expriment leurs sincères remerciements aux tradithérapeutes de l'Association Nationale des Praticiens de la Médecine Traditionnelle du Bénin (ANaPraMeTrab) qui ont participé activement à cette étude.

\section{REFERENCES}

Abdulaziz R, Ibrahim U, Tambari B, Nafiu A, Jumare I, Said M, Ibrahim AD. 2019. Studies on the Antibacterial Activity and Chemical Composition of Methanol Extract of Cochlospermum tinctorium Root. Asian Plant Research Journal, 2(3): $\quad 1-11 . \quad$ DOI: https://doi.org/10.9734/aprj/2019/v2i330 049

Adomou AC, Agbani OP, Sinsin B. 2011. Plantes. In Protection de la Nature en Afrique de l'Ouest: Une Liste Rouge pour le Bénin. Nature Conservation in West Africa: Red List for Benin. International Institute of Tropical Agriculture; 21-46.

Agbodjento E, Klotoé JR, Sacramento TI, Dougnon V, Tchabi FL, Déguénon E, Atègbo JM. 2020. Ethnobotanical knowledge of medicinal plants used in the treatment of male infertility in southern Benin. Adv. Tradit. Med. (ADTM). https://doi.org/10.1007/s13596-02000473-3

Ahmad MH, Jatau AI, Khalid GM, Alshargi OY. 2021. Traditional uses, phytochemistry, and pharmacological activities of Cochlospermum tinctorium A. Rich (Cochlospermaceae): a review. Futur J Pharm Sci, 7(1): 20. DOI: https://doi.org/10.1186/s43094-02000168-1

Aissi AK, Kougblenou S, Dougnon V, Klotoe JR, Bankole H, Deguenon Y, Degbey C, Montcho S, Fanou B, Fah L, Edorh P, Loko F. 2013. Evaluation de la qualité sanitaire des poudres de feuilles de Moringa oleifera Lam. commercialisées au profit des Personnes Vivant avec le VIH à Cotonou (Bénin). Int. J. Biol. Chem. Sci, 7(4): 1461-1473.

Akoègninou A. 2004. Recherches botaniques et écologiques sur les forêts actuelles du Bénin. (Thèse d'Etat), Université de Cocody-Abidjan, Côte d'Ivoire, p. 326.

Akpemi A. 2012. Cytotoxicity Activity and Phytochemical Screening of Cochlospermum tinctorium Perr Ex A. Rich Rhizome. Journal of Applied Pharmaceutical Science, 2(7): 155-159.

Ballin NZ, Traore M, Tinto H, Sittie A, Mølgaard P, Olsen CE, Kharazmi A, Christensen SB. 2002. Antiplasmodial Compounds from Cochlospermum tinctorium. J. Nat. Prod, 65(9): 13251327.

DOI: https://doi.org/10.1021/np020008h

Benkhnigue O, Zidane L, Fadli M, Elyacoubi H, Rochdi A, Douira A. 2010. Etude ethnobotanique des plantes médicinales dans la région de Mechraâ Bel Ksiri (Région du Gharb du Maroc). Acta Botanica Barcinonensia, 53:191-216.

El Yahyaoui O, Ait ouaaziz N, Sammama A, Kerrouri S, Bouabid B, Lrhorfi L, Zidane L, Bengueddour R. 2015. Etude ethnobotanique: Plantes médicinales commercialisées à la province de Laâyoune; identification et utilisation. International Journal of Innovation and Applied Studies, 12(3): 533-541.

FAO-WHO. 2020. Normes officielles | CODEXALIMENTARIUS FAO-WHO 
[WWW Document]. URL http://www.fao.org/fao-whocodexalimentarius/codex-texts/liststandards/fr/ (accessed 12.21.20).

Favi GA, Dassou GH, Adomou AC, Fandohan AB, Sogbohossou EOD, Yedomonhan H, Tossou MG, Akoègninou A. 2020. Cochlospermum planchonii Hook.f. ex Planch. and Cochlospermum tinctorium Perrier ex A. Rich.: extent of knowledge and prospects for sustainable use in West Africa. Genet Resour Crop Evol, 68: 2544. DOI: https://doi.org/10.1007/s10722020-01062-8

Hachi M, Hachi T, Belahbib N, Dahmani J, Zidane L. 2015. Contribution à l'etude floristique et ethnobotanique de la flore médicinale utilisée au niveau de la ville de khenifra (maroc). International Journal of Innovation and Applied Studies, 11(3): 754-770.

Igoli JO, Igwue IC, Igoli NP. 2004. Traditional Medicinal Practices Among the Igede People of Nigeria. Journal of Herbs, Spices \& Medicinal Plants, 10(4): 1-10. DOI:

https://doi.org/10.1300/J044v10n04_01

Igue AM, Saidou A, Adjanohoun A, Ezui G, Attiogbe P, Kpagbin G, GotoechanHodonou H, Youl S, Pare T, Balogoun I, Ouedraogo J, Dossa E, Mando A, Sogbedji JM. 2013. Evaluation de la fertilité des sols au sud et centre du Bénin. Bulletin de la Recherche Agronomique du Bénin: 12-23.

INSAE. 2013. Enquête Démographique générale 2013. Ministère de la Prospective du Développement et de l'Evaluation de l'Action Publique, Institut National de la Statistique et de l'Analyse Economique. Republique du Bénin (Cotonou).

Klotoé JR, Dougnon TV, Ategbo JM, Koudokpon H, Dramane K. 2013. Ethnopharmacological Survey on Antihemorrhagic Medicinal Plants in South of Benin. European Journal of Medicinal Plants, 3(1): 40-51. DOI: https://doi.org/10.9734/EJMP/2013/2093

Maiha vii, Magaji M, Yaro A, Hamza Magaji, R. 2009. Anticovulsant Studies on
Cochlospermum tinctorium (Cochlospermaceae) and Paullinia pinnata (Sapindaceae) Extracts in Laboratory Animals. Nigerian Journal of Pharmaceutical Sciences, 8(1): 102-108.

Mehdioui R, kahouadji A. 2007. Etude ethnobotanique auprès de la population riveraine de la forêt d'Amsittène: cas de la Commune d'Imi n'Tlit (Province d'Essaouira). Bulletin de l'Institut Scientifique, 29: 11-20.

Mouzouvi R, Djégo JG, Lalèyè A, Priuli F, Bigot A. 2014. Effet de l'association Combretum micranthum G. Don (Combretaceae) et Cochlospermum tinctorium A. Rich. (Cochlospermaceae) dans la prise en charge de l'hépatite virale B. Pharmacopée et Médecine Traditionnelle Africaine, 17(1): 10-14.

Nergard C, Diallo D, Inngjerdingen K, Michaelsen T, Matsumoto T, Kiyohara $\mathrm{H}$. 2005. Medicinal use of Cochlospermum tinctorium in Mali, Anti-ulcer, radical scavenging and immunomodulating activites of polymers in the aqueous extracts of the roots. J. Ethnopharmacol, 96(1-2): 255-269.

OMS. 2002. Stratégie de l'OMS pour la Médecine Traditionnelle pour 20022005, Genève.

Tabuti JRS, Lye KA, Dhillion SS. 2003. Traditional herbal drugs of Bulamogi, Uganda: plants, use and administration. $J$ Ethnopharmacol, 88(1): 19-44. DOI: https://doi.org/10.1016/s03788741(03)00161-2

The Plant List. 2012. Cochlospermum tinctorium Perrier ex A.Rich. - The Plant List [WWW Document]. URL http://www.theplantlist.org/tpl1.1/record/ kew-44552 (accessed 5.31.21).

Zerbo P, Millogo-Rasolodimey J, NacoulmaOuerdraogo OG, Damme PV. 2007. Contribution à la connaissance des plantes médicinales utilisées dans les soins infantiles en pays San, au Burkina Faso. International Journal of Biological and Chemical Sciences, 1(3): 262-274. DOI:

https://doi.org/10.4314/ijbcs.v1i3.39704 